\title{
The effect of active and passive maternal smoking before and during pregnancy on neonatal weight at birth
}

Cezary Wojtyla ${ }^{1,2}$, Paulina Wojtyla-Buciora ${ }^{3,4}$, Michal Ciebiera $^{5}$, Stanisław Orzechowski ${ }^{6}$, Andrzej Wojtyla ${ }^{3}$

\author{
${ }^{1}$ European Observatory of Health Inequalities, State University of Applied Sciences, \\ Kalisz, Poland \\ ${ }^{2}$ Department of Oncological Gynecology and Obstetrics, Centre of Postgraduate \\ Medical Education, Warsaw, Poland \\ ${ }^{3}$ Faculty of Medicine, State University of Applied Sciences, Kalisz, Poland \\ ${ }^{4}$ Department of Physiology, University of Medical Sciences, Poznan, Poland \\ ${ }^{5}$ Second Department of Obstetrics and Gynecology, Centre of Postgraduate Medical \\ Education, Warsaw, Poland \\ ${ }^{6}$ Department of Nursing, Faculty of Health Sciences, University of Warmia and \\ Mazury, Olsztyn, Poland
}

Submitted: 20 November 2017; Accepted: 17 January 2018;

Online publication: 15 November 2018

Arch Med Sci 2021; 17 (2): 352-360

DOI: https://doi.org/10.5114/aoms.2018.79629

Copyright @ 2018 Termedia \& Banach

\begin{abstract}
Introduction: Smoking during pregnancy is a risk factor for adverse pregnancy outcomes. Data on the correlation between passive maternal smoking and pregnancy outcomes remain limited. We investigated the effect of active smoking and environmental tobacco smoke (ETS) during pregnancy on neonatal birthweight, including the risk for low birthweight (LBW).

Material and methods: The study was conducted between 2010 and 2012. A group of 8625 women were surveyed during postpartum hospitalization. Outcome measures included mean birthweight of newborns. Additionally, odds ratios with confidence intervals were calculated to investigate the risk for LBW in active and passive smoking groups of mothers.

Results: Lower birthweight (46 g- $307 \mathrm{~g} ; p<0.05)$ and a higher risk for LBW $(\mathrm{OR}=1.35,95 \% \mathrm{Cl}: 1.05-1.75 ; p<0.05)$ were observed in all infants born to smoking mothers. A negative effect of ETS in pregnancy on the reduction of mean birthweight was also found. Additionally, we analyzed the cumulative effect of active and passive smoking on neonatal birthweight. A statistically significant reduction in neonatal weight at birth was found in a group of women who smoked actively and passively during pregnancy (130 g; $p<0.05$ ). Conclusions: Smoking is associated with decreased birthweight and in a group of active smoking mothers increased risk for LBW. This effect is dose-dependent and is also present in a group of women who smoked before pregnancy. There is also a cumulative effect of active smoking and ETS causing decreased neonatal birthweight and increased risk for low birthweight.
\end{abstract}

Key words: tobacco, environmental tobacco smoke, maternal smoking, birthweight.

\section{Introduction}

Active smoking in pregnancy is believed to be the key modifiable risk factor for low birthweight (LBW), i.e. < 2500 g. At present, LBW constitutes one of the main causes of neonatal mortality [1], as well as chronic diseases, in adult life [2, 3], in the developed countries. The risk for adverse pregnancy outcomes associated with active smoking is correlat-

\author{
Corresponding author: \\ Cezary Wojtyla \\ Department \\ of Oncological \\ Gynecology \\ and Obstetrics \\ Centre of \\ Postgraduate \\ Medical Education \\ 231 Czerniakowska St \\ 00-416 Warsaw, Poland \\ Phone: +48 225841160 \\ E-mail: czwo@op.pl
}


ed with the number of cigarettes smoked per day and duration of smoking during pregnancy [4-7]. Passive maternal smoking, also known as 'secondhand smoke' or 'environmental tobacco smoke' (ETS), is defined as exposure to tobacco smoke exhaled by a smoker (mainstream smoke) and smoke which wafts off the end of the lit cigarette (side-stream smoke) [8]. The concentration of harmful toxins contained in tobacco smoke, especially side-stream smoke, is believed to exceed their level in the smoke inhaled by a smoker [911]. Thus, a passive smoker is exposed to virtually the same substances as an active smoker, which include tar, nicotine, carbon dioxide, carbon monoxide, and polycyclic aromatic hydrocarbons [12]. Approximately $22-30 \%$ of pregnant non-smokers have been estimated to be exposed to ETS [13]. The mechanism of the potential influence of ETS on the developing fetus remains the subject of extensive research [14-17]. The negative effects of active smoking during pregnancy on LBW are no longer questioned but data on the correlation between ETS and pregnancy outcomes, including neonatal weight, remain limited.

The aim of the study was to investigate the effects of active smoking before and during various trimesters of pregnancy and frequency of exposure to ETS during pregnancy on neonatal birthweight, including the risk for LBW.

\section{Material and methods}

The analysis was conducted using survey questionnaires within the Polish Pregnancy-related Assessment Monitoring System (Pol-PrAMS). The methodology of this study and information about Pol-PrAMS are described in detail in a separate article [18]. This population-based study was carried out between 2010 and 2012 in all public hospitals in Poland (373, 379 and 377 hospitals in 2010, 2011 and 2012 respectively). Permission to carry out the survey had been received from the director of each hospital.

A group of Polish women and their newborns were investigated during postpartum hospitalization (first days after delivery). All women in Poland who stayed in those public hospitals whose director gave permission to carry out the survey were deemed eligible for the study. Informed consent was obtained from all women. Participation was voluntary. Thus, the study participants were those women who voluntarily agreed to join the study from all women who stayed in researched public hospitals in Poland during designated days of the study. Overall, 9051 mothers and their newborns were hospitalized in obstetric wards of researched hospitals. Out of them, 8625 (95.3\%) mothers who agreed to join the study and answered at least one question in the survey were included in the study. In non-researched hospi- tals there were at that time 2091 postpartum women. These were non-public hospitals or hospitals whose directors did not agree to conduct the study. Figure 1 shows exclusion and inclusion criteria.

The Local Ethics Committee, a body within the Institute of Rural Health in Lublin, approved of the study (reference number 03/2011). The survey included questions about the number of smoked cigarettes in the following periods: last 3 months before pregnancy, first 3 months of pregnancy, last 3 months before delivery, and at present, i.e. first few days after delivery. Passive smoking during pregnancy, i.e. frequency of exposure to tobacco smoke at home and at work, was also investigated.

\section{Statistical analysis}

Analysis of data was performed using IBM SPSS (V22) statistical software. The $\chi^{2}$ test was used to analyze the dependency between categorical variables. Odds ratios (OR) with confidence intervals (CI) were calculated to analyze the risk for LBW according to active maternal smoking and environmental tobacco smoke. The $p$-value of $<0.05$ for a two-tailed test was considered as statistically significant. We analyzed such variables as age, height, weight, place of residence, type of work, material status, social conditions, active and passive smoking status, neonatal weight and risk for LBW. Due to the fact that not all questions were completed, the sample size in the description of the questions is smaller than the actual number of women deemed eligible for the study, so the percentage value is the so-called valid percent, calculated for the number of answers and not the number of completed questionnaires.

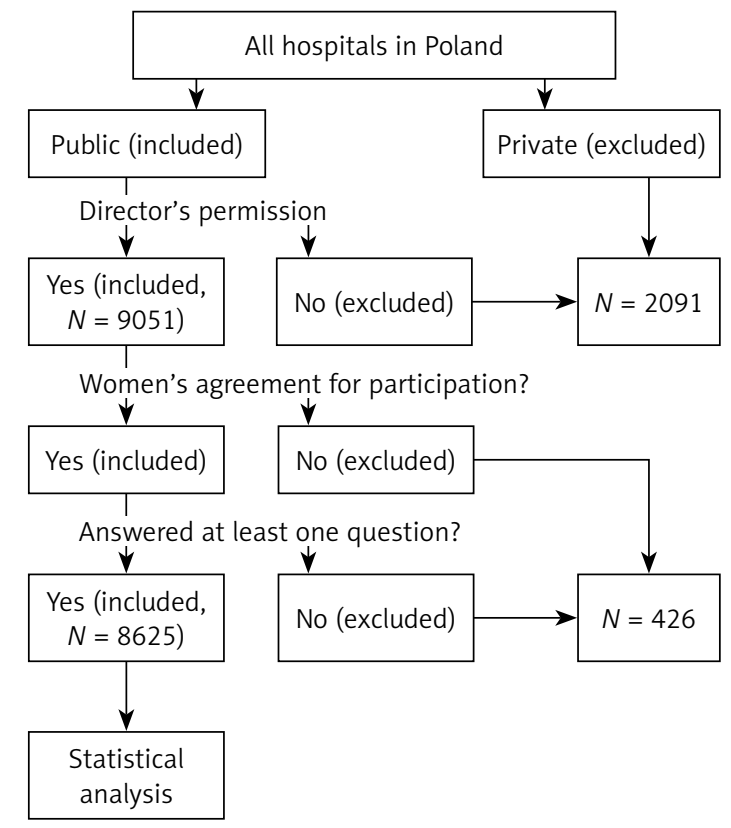

Figure 1. Exclusion and inclusion criteria 


\section{Results}

Patient characteristics are presented in Table I. Median age and height of the subjects deemed eligible for the study were 28.3 years and $1.65 \mathrm{~m}$, respectively. Median pre-pregnancy and pre-delivery weight was $60.0 \mathrm{~kg}$ and $75.5 \mathrm{~kg}$, respectively. Intellectual workers constituted the largest (42.9\%) group of women, physical workers and unemployed rates were $26.2 \%$ and $25.4 \%$, respectively, while students comprised $5.6 \%$ of the study group. The majority of the women were city residents but over $42 \%$ inhabited rural areas. Over half of the respondents declared their economical status and social conditions to be good.

Active smoking in pregnancy by participants of the Pol-PrAMS study is presented in another paper [19]. A total of $18.1 \%$ of the women reported smoking in the last 3 months before pregnancy, while $81.9 \%$ reported no smoking during that period. The rates of women smoking at least 1 cigarette in the first 3 months of pregnancy and the last trimester were $6.8 \%$ and $7.7 \%$, respectively, whereas $3.6 \%$ of the respondents reported smoking after delivery. The home environment was the main source of ETS exposure for the pregnant women. Only a little over $60 \%$ and almost $80 \%$ of the women reported absence of active smokers at their home and work, respectively. The number and percentage of women smoking cigarettes and their exposure to ETS are presented in Table II.

A statistically significantly lower birthweight was observed for all periods of active smoking in all infants born to smoking as compared to non-smoking mothers. The latter gave birth to children with a mean birthweight of $3374.74 \mathrm{~g}$. Smoking resulted in decreased neonatal weight at birth.
Thus, infants born to mothers who smoked $>10$ cigarettes a day were $105 \mathrm{~g}$ lighter. The difference in mean birthweight of neonates born to mothers who smoked in the first 3 months of pregnancy was $108.58 \mathrm{~g}$ as compared to non-smoking mothers. The greatest difference was noted among women who smoked in the third trimester of pregnancy. The difference between non-smoking mothers and those who smoked over half a pack of cigarettes a day was 307.06 g. Also, lower birthweight was observed in children born to mothers who smoked after delivery. The difference in birthweight of children born to mothers who did not smoke and those who smoked $>10$ cigarettes a day was $204.98 \mathrm{~g}$.

Women who were not exposed to ETS at home gave birth to children with a mean birthweight of $3388.21 \mathrm{~g}$. An increasing frequency of exposure to tobacco smoke at home was connected with a gradual decrease in neonatal birthweight. Thus, women exposed to tobacco smoke at home on a daily basis gave birth to children with a mean birthweight decreased by $103.69 \mathrm{~g}$. Also, lower neonatal birthweight (by $78.32 \mathrm{~g}$ ) was found in the group of mothers who were exposed to ETS at work on a daily basis, with the one exception of women exposed to ETS at work several times a month, who gave birth to infants with higher birthweight (by $36.31 \mathrm{~g}$ ) as compared to mothers not exposed to ETS at work.

Additionally, we analyzed the cumulative effect of active and passive smoking on neonatal birthweight. Women who did not smoke during pregnancy and were not exposed to ETS at home gave birth to children with a mean birthweight of 3392.85 g. Children born to mothers who did

Table I. Patient characteristics

\begin{tabular}{|c|c|c|c|c|c|}
\hline Parameter & Median & SD & Minimum & Maximum & \\
\hline Age [years] & 28.3 & 5.13 & 14 & 51 & \\
\hline Height [m] & 1.65 & 0.09 & 0.0 & 1.94 & \\
\hline \multicolumn{6}{|l|}{ Weight [kg]: } \\
\hline Pre-pregnancy & 60.0 & 12.14 & 33.0 & 169.0 & \\
\hline Pre-delivery & 75.5 & 12.80 & 33.0 & 175.0 & \\
\hline Place of residence & City > 500000 & $100-500000$ & $50-100000$ & $<50000$ & Rural area \\
\hline Value & $11.2 \%$ & $13.4 \%$ & $11.1 \%$ & $21.8 \%$ & $42.4 \%$ \\
\hline Type of work & Intellectual & Physical & Unemployed & Student & \\
\hline Value & $42.9 \%$ & $26.2 \%$ & $25.4 \%$ & $5.6 \%$ & \\
\hline Parameter & Very good & Good & Average & Bad & \\
\hline Economic status & $9.5 \%$ & $56.5 \%$ & $32.6 \%$ & $1.4 \%$ & \\
\hline Social conditions & $24.1 \%$ & $56.4 \%$ & $19 \%$ & $0.5 \%$ & \\
\hline
\end{tabular}


not smoke actively during pregnancy but were exposed to ETS at home reached a mean birthweight of $3341.53 \mathrm{~g}$, whereas some women who reported active and passive smoking during pregnancy gave birth to children with a mean birthweight of 3262.62 g. All data were statistically significant and the results are presented in Table III.

Active smoking at any stage of pregnancy was associated with the risk for LBW (OR $=1.35$, $95 \% \mathrm{Cl}$ : 1.05-1.75). Such risk was not observed in the group of women who smoked only before pregnancy and in the first 3 months of pregnancy. The odds ratio for LBW in the group of women who smoked in the last 3 months of pregnancy was higher as compared to non-smokers in pregnancy $(\mathrm{OR}=1.63,95 \% \mathrm{Cl}: 1.23-2.16)$. The highest risk for LBW infants was noted among women who reported smoking after delivery $(\mathrm{OR}=1.97,95 \% \mathrm{Cl}$ : 1.36-2.87). The connection between ETS at home and the risk for LBW was not statistically significant $(\mathrm{OR}=1.17,95 \% \mathrm{Cl}:$ 0.98-1.41). That risk was not observed among women exposed to ETS at work. No statistically significantly higher risk for LBW was found among women who did not smoke actively but were exposed to passive smoking at home, contrary to women who smoked actively during pregnancy and were additionally exposed to ETS at home $(\mathrm{OR}=1.46,95 \% \mathrm{Cl}: 1.10-1.96)$. Table IV shows odds ratios (ORs) and 95\% confidence intervals (Cls) for LBW according to active maternal smoking and environmental tobacco smoke during pregnancy.

\section{Discussion}

The present study has confirmed a link between active smoking in pregnancy and lower birthweight, as well as the risk for LBW infants. The correlation was dose-dependent: the more cigarettes the woman smoked before pregnancy, the lower the neonatal weight at birth. It seems that smoking in the last 3 months of pregnancy has the most detrimental effect on mean neonatal birthweight. Also, the odds ratio for LBW infants was higher among women who smoked in the last trimester than at any time in pregnancy, but the highest risk was noted for women who smoked after delivery, possibly due to the fact that they were the most addicted to nicotine and smoked throughout the entire course of pregnancy. A negative effect of ETS in pregnancy on the reduction of birthweight was also found.

The cumulative effect of active and passive smoking on neonatal birthweight was also analyzed. A statistically significant reduction in newborn weight at birth was found in active and passive smokers during pregnancy. The difference was more visible as compared to women who were non-active smokers but were exposed to ETS at home. In comparison to all of the above-
Table II. Number and percentage of women smoking cigarettes before and during pregnancy [19] and their exposure to ETS during pregnancy

\begin{tabular}{|c|c|c|}
\hline Variable & $N$ & $\%$ \\
\hline \multicolumn{3}{|l|}{ Active smoking: } \\
\hline 3 months before pregnancy: & 8578 & 100.0 \\
\hline None & 7022 & 81.9 \\
\hline $1-10$ & 1055 & 12.3 \\
\hline$>10$ & 501 & 5.8 \\
\hline First 3 months of pregnancy: & 8553 & 100.0 \\
\hline None & 7965 & 93.2 \\
\hline $1-10$ & 508 & 5.9 \\
\hline$>10$ & 80 & 0.9 \\
\hline Last 3 months of pregnancy: & 8595 & 100.0 \\
\hline None & 7936 & 92.3 \\
\hline $1-10$ & 577 & 6.7 \\
\hline$>10$ & 82 & 1.0 \\
\hline First few days after delivery: & 8590 & 100.0 \\
\hline None & 8284 & 96.4 \\
\hline $1-10$ & 260 & 3.0 \\
\hline$>10$ & 46 & 0.6 \\
\hline \multicolumn{3}{|l|}{ ETS } \\
\hline At home: & 8375 & 100.0 \\
\hline Never & 5086 & 60.7 \\
\hline Several times a month & 845 & 10.1 \\
\hline Several times a week & 552 & 6.6 \\
\hline Every day & 1892 & 22.6 \\
\hline At work: & 7507 & 100.0 \\
\hline Never & 5981 & 79.7 \\
\hline Several times a month & 312 & 4.2 \\
\hline Several times a week & 289 & 3.8 \\
\hline Every day & 925 & 12.3 \\
\hline Active smoking and ETS: & 7484 & 100.0 \\
\hline Active $(-)^{b}$; ETS at home $(-)^{c}$ & 4499 & 60.1 \\
\hline Active $(-)$; ETS at home $(+)^{d}$ & 2269 & 30.3 \\
\hline Active $(+)^{\mathrm{e}}$; ETS at home $(+)$ & 716 & 9.6 \\
\hline
\end{tabular}

aEnvironmental tobacco smoke, ${ }^{b}$ active (-) - non-smokers during pregnancy, ' ${ }^{C}$ TS at home (-) - not exposed to environmental tobacco smoke at home, ${ }^{d} E T S$ at home (+) - exposed to environmental tobacco smoke at home during pregnancy, eactive (+) - active smokers during pregnancy.

mentioned groups, pregnant women who did not smoke, either actively or passively, gave birth to infants with the highest birthweight. 
Table III. Median and mean neonatal weight versus the number of smoked cigarettes before and during pregnancy and frequency of exposure to ETS during pregnancy

\begin{tabular}{|c|c|c|c|c|c|}
\hline Variable & Mean & Mean difference & SD & Median & $P$-value \\
\hline \multicolumn{6}{|l|}{ Active smoking: } \\
\hline 3 months before pregnancy: & & & & & $<0.05$ \\
\hline None & 3374.74 & 0 & 570.48 & 3400.0 & \\
\hline $1-10$ & 3328.55 & -46.19 & 557.88 & 3370.0 & \\
\hline$>10$ & 3269.74 & -105.0 & 543.04 & 3300.0 & \\
\hline First 3 months of pregnancy: & & & & & $<0.05$ \\
\hline None & 3367.75 & 0 & 569.27 & 3400.0 & \\
\hline $1-10$ & 3312.87 & -54.88 & 552.72 & 3350.0 & \\
\hline$>10$ & 3259.17 & -108.58 & 490.68 & 3200.0 & \\
\hline Last 3 months of pregnancy: & & & & & $<0.05$ \\
\hline None & 3374.94 & 0 & 566.78 & 3400.0 & \\
\hline $1-10$ & 3236.31 & -138.63 & 559.09 & 3285.0 & \\
\hline$>10$ & 3067.88 & -307.06 & 567.59 & 3000.0 & \\
\hline At present: & & & & & $<0.05$ \\
\hline None & 3369.27 & 0 & 567.17 & 3400.0 & \\
\hline $1-10$ & 3195.02 & -174.25 & 560.73 & 3230.0 & \\
\hline$>10$ & 3164.29 & -204.98 & 598.25 & 3185.0 & \\
\hline \multicolumn{6}{|l|}{ ETS: } \\
\hline At home: & & & & & $<0.05$ \\
\hline Never & 3388.21 & 0 & 569.61 & 3410.0 & \\
\hline Several times a month & 3369.79 & -18.47 & 568.10 & 3420.0 & \\
\hline Several times a week & 3363.95 & -24.28 & 533.83 & 3390.0 & \\
\hline Every day & 3284.52 & -103.69 & 563.44 & 3320.0 & \\
\hline At work: & & & & & $<0.05$ \\
\hline Never & 3376.06 & 0 & 570.52 & 3400.0 & \\
\hline Several times a month & 3412.37 & 36.31 & 537.05 & 3430.0 & \\
\hline Several times a week & 3343.73 & -32.33 & 575.82 & 3370.0 & \\
\hline Every day & 3297.74 & -78.32 & 560.55 & 3340.0 & \\
\hline Active smoking and ETS: & & & & & $<0.05$ \\
\hline Active $(-)^{a}$; ETS at home $(-)^{b}$ & 3392.85 & 0 & 568.37 & 3420.0 & \\
\hline Active $(-)$; ETS at home $(+)^{c}$ & 3341.53 & -51.32 & 563.08 & 3390.0 & \\
\hline Active $(+)^{\text {d }}$; ETS at home $(+)$ & 3262.62 & -130.23 & 557.87 & 3300.0 & \\
\hline
\end{tabular}

Our findings are consistent with earlier reports on the negative effects of active and passive maternal smoking during pregnancy on neonatal weight at birth. The birthweight of children born to active smokers during pregnancy has been es- timated to be $150-250 \mathrm{~g}$ lower as compared to pregnant non-smokers. Also, the risk for LBW has been reported [20-23]. The correlation between active maternal smoking and neonatal birthweight depends on the number of cigarettes a day and is 
Table IV. Odds ratios and $95 \%$ confidence intervals for low birthweight according to active maternal smoking and environmental tobacco smoke before and during pregnancy

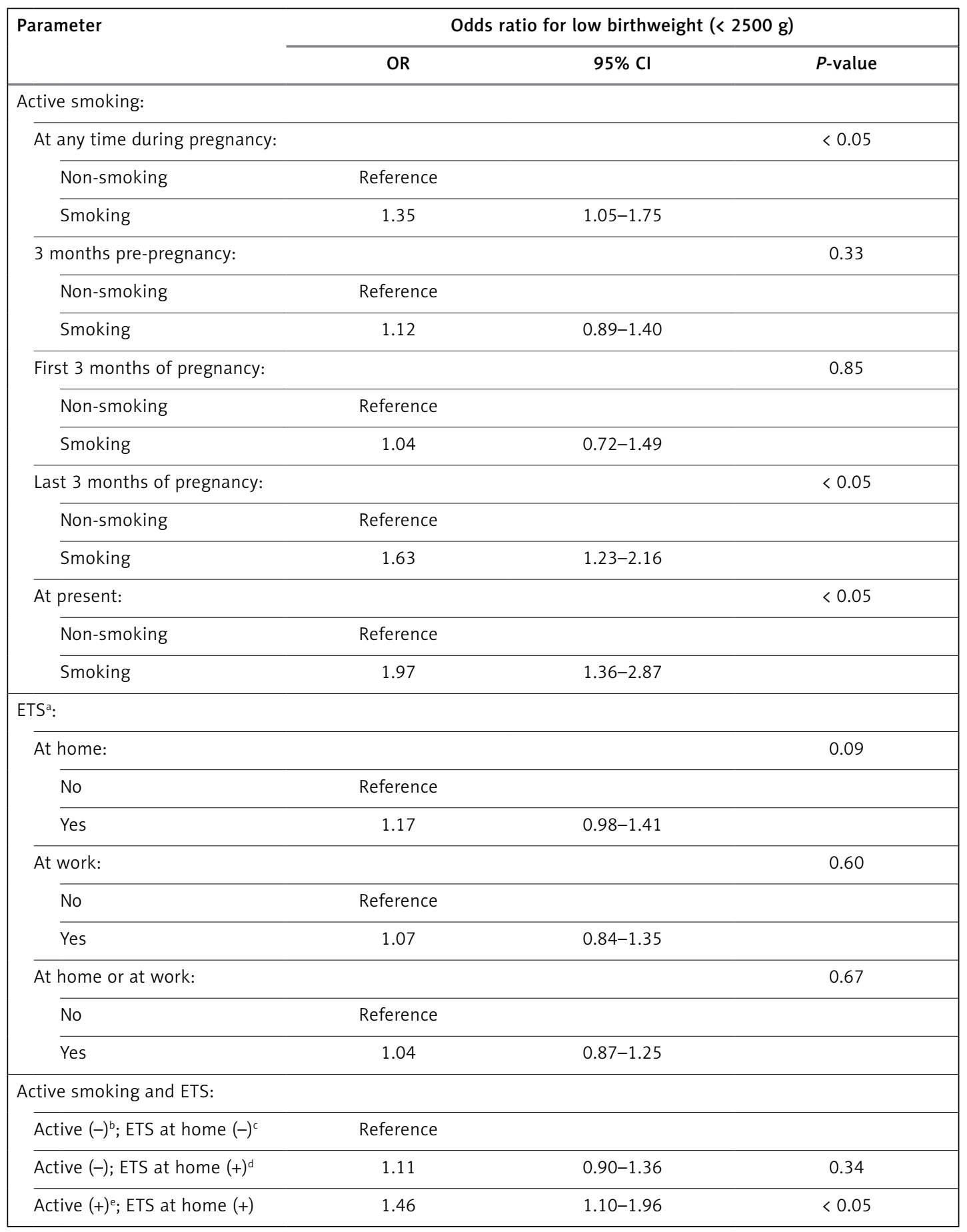

${ }^{a}$ Environmental tobacco smoke, ${ }^{b}$ active (-) - non-smokers during pregnancy, ${ }^{c} E T S$ at home (-) - not exposed to environmental tobacco smoke at home, ${ }^{d} E T S$ at home (+) - exposed to environmental tobacco smoke at home during pregnancy, ${ }^{e}$ active (+) - active smokers during pregnancy.

visible even for the lowest amount of tobacco in active smokers $[6,24]$. It seems that smoking only in the first 3 months of pregnancy does not negatively affect neonatal birthweight or the risk for LBW. Research shows that the birthweight values of children born to mothers who quit smoking in the first 3 months of pregnancy and refrained from smoking until delivery versus mothers who never smoked were comparable [21, 22, 25]. Our results indicate that birthweight of infants born to mothers who smoked in the first 3 months of pregnancy was significantly lower as compared to mothers who did not smoke during that period. We did not detect elevated risk for LBW in that group of wom- 
en. Importantly, we did not investigate whether these subjects continued to smoke throughout the remaining stages of pregnancy or quit smoking. It seems that birthweight of children born to mothers who limited the number of cigarettes during pregnancy is higher as compared to mothers who continued to smoke in pregnancy, but lower as compared to non-smokers [21]. Without a doubt, smoking in the third trimester had the greatest influence on neonatal birthweight. Children born to mothers from that group were the lightest [24], which is consistent with our findings.

Bernstein et al. are of the opinion that prepregnancy smoking has no influence on neonatal birthweight [24], which is in contrast to our observations. In our study, mean birthweight of children born to women who smoked before pregnancy was as much as $105 \mathrm{~g}$ lighter than children of non-smoking women. Neonatal birthweight in that group of children was dose-dependent. It was correlated with the number of smoked cigarettes.

It appears that not only active smoking has a negative effect on neonatal weight at birth. The study with a meta-analysis by Salmasi et al. demonstrated that ETS during pregnancy was connected with birthweight reduction by $60 \mathrm{~g}$ [8]. Two other meta-analyses also reported reduced birthweight in children born to passive smokers in pregnancy but the difference was smaller by half $[11,26]$. Also, one of them, the study by Leonardi-Bee et al., demonstrated an elevated risk for LBW among those women $(\mathrm{OR}=1.32 ; 95 \% \mathrm{Cl}$ : $1.07-1.63$ in prospective and $\mathrm{OR}=1.22 ; 95 \% \mathrm{Cl}$ : $1.08-1.37$ in retrospective studies) [26], which is consistent with our findings. The difference between birthweight of children born to mothers exposed to ETS at home was $103 \mathrm{~g}$. The difference between birthweight of children born to mothers exposed to ETS at work was $78 \mathrm{~g}$. Both of them were dose-dependent. Jaddoe et al. found no correlation between the effects of passive smoking during early pregnancy and reduced neonatal birthweight or risk for LBW. Passive smoking of more than $3 \mathrm{~h}$ a day, in late pregnancy, was associated with significantly elevated risk for LBW but not mean neonatal weight [21]. Also, no link between a changed number of ETS hours and mean neonatal weight at birth was observed, while the effect of ETS on birthweight was uncertain [21].

Pogodina et al. found a correlation between ETS and the risk for LBW children [27]. They reported the risk to be increasing with the number of active smokers around a pregnant woman, which is consistent with our findings of a correlation between ETS dose and birthweight. Notably, the methods of measuring ETS doses differ between the studies. Jaddoe et al. evaluated the dose on the ba- sis of the number of ETS-exposure hours during a day [21], while Pogodina et al. used the number of active smokers at home of a pregnant woman [27]. In our study, the frequency of smoking in the presence of a pregnant woman at home and at work was used as the measuring factor. Thus, in light of the discrepancies in methodology, the results of various studies ought to be compared with caution. We believe that the question used in our study has the advantage over the question about the number of active smokers at home of a pregnant woman due to the fact that these people do not necessarily smoke in her presence or may smoke outside.

Pogodina et al. investigated the cumulative effect of active and passive smoking on the risk for LBW [27], and found it to be identical for active and passive smokers, active but not passive smokers, as well as non-smokers. Only non-smokers who were exposed to ETS were found to be at higher risk for $\mathrm{LBW}(\mathrm{OR}=1.15 ; 95 \% \mathrm{Cl}$ : 0.91-1.44). In our study, we observed a cumulative effect of active and passive smoking in pregnancy on the reduction of mean neonatal weight at birth. Women who were neither active nor passive smokers during pregnancy gave birth to children with the highest weight, and infants born to mothers exposed to ETS at home but who refrained from active smoking were $51 \mathrm{~g}$ lighter. The birthweight of neonates born to mothers who smoked in pregnancy (actively and passively) was $130 \mathrm{~g}$ lower. In the analysis of the cumulative effect of active and passive smoking in pregnancy, we included only ETS at home as it is the main place of ETS exposure for non-smoking adults [28]. We observed an elevated risk for LBW among mothers who smoked, both actively and passively, during pregnancy.

Our study is not without limitations. As a survey questionnaire with a self-declared number of smoked cigarettes a day and exposure to ETS was used, it is not possible to exclude recall bias. Furthermore, the results of our study have not been validated using the method of biochemical markers, e.g. cotinine in urine, saliva or hair, so misclassification bias cannot be excluded, either. Regardless, reports from England et al. and Haddow et al. did not confirm the superiority of methodology based on biochemical markers for studies on tobacco smoke and neonatal weight at birth [6, 29]. Additionally, Pickett et al. noted that the number of cigarettes a day determined in survey questionnaires and cotinine levels in urine are highly correlated at any stage of pregnancy [30]. Salmasi et al., in their study with a meta-analysis on ETS and neonatal birthweight, found no differences in the final results between self-reported questionnaires and biochemical markers. What is 
more, they also detected no differences between retrospective and prospective studies [8]. Other studies with meta-analyses generated similar results [26]. The main limitation of our study was lack of control for confounding variables such as maternal age, parity, socioeconomic status, alcohol or drug consumption, and neonatal sex. Furthermore, we were not able to objectively evaluate the exact level of ETS exposure. As mentioned above, the home environment is the main source of ETS for non-smoking children and adults [28], and that type of exposure was used to assess the cumulative effect of active and passive smoking on neonatal birthweight. Additionally, exposure to ETS at work was also investigated. ETS exposure at home and at work was evaluated separately, without their cumulative effect on neonatal birthweight. Furthermore, pregnant women may be at risk for ETS elsewhere, e.g. homes of their friends or family, not to mention public places. Also, it is important to consider other uncontrolled confounding variables which resulted from our survey questions. We lack knowledge about the number of hours of ETS exposure, the number of smokers, and the number of cigarettes smoked in the presence of a pregnant woman. An analysis of these variables might affect the results. We included only women who smoked, or did not smoke actively, any number of cigarettes at any stage of pregnancy, and those exposed to ETS of any frequency in our assessment of the cumulative effect of active and passive smoking. Possibly, different criteria, e.g. active smoking throughout the entire pregnancy or daily exposure to ETS, might have resulted in greater LBW risk and additional reduction of mean neonatal weight at birth. Our study was carried out in public hospitals in Poland. It did not include women who stayed in private hospitals or in hospitals whose directors did not agree to take part in the study. Thus, we cannot generalize our findings to the whole population of pregnant women.

The strength of our study included its population character. The study was conducted in all public hospitals in Poland which provided care for parturients; thus we minimized selection bias. Both exposure at home and at work were assessed, thus allowing us to identify the main source of ETS exposure. Most studies on the effects of ETS on pregnancy outcomes evaluated only ETS at home, using different methodology [26]. Furthermore, our questions focused on the frequency of other people smoking in the presence of a pregnant woman, allowing the risk for exposure at home or at work to be established with great precision. Finally, our methodology allowed for the analysis of the cumulative effect of active and passive smoking in pregnancy.
In conclusion, active and passive smoking in pregnancy is associated with significantly decreased mean birthweight and in a group of active smoking mothers increased risk for LBW. This effect is dose-dependent and is also present in a group of women who smoked before pregnancy. There is also a cumulative effect of active smoking and environmental tobacco smoke causing decreased neonatal birthweight and increased risk for LBW. An important aspect of this study is the finding of a negative impact not only of active but also passive smoking during pregnancy on neonatal birthweight and the increased risk for LBW. Pregnant women should be aware of this impact and avoid both sources of tobacco exposure.

\section{Conflict of interest}

The authors declare no conflict of interest.

\section{References}

1. Lawn JE, Cousens S, Zupan J; Neonatal Survival Steering Team. 4 million neonatal deaths: when? Where? Why? Lancet 2005; 365: 891-900.

2. Walsh RA. Effects of maternal smoking on adverse pregnancy outcomes: examination of the criteria of causation. Hum Biol 1994; 66: 1059-92.

3. Barker DJ. Fetal origins of coronary heart disease. BMJ 1995; 311: 171-74.

4. Lindsay J, Royle C, Heaman M. Rate of Maternal Smoking During Pregnancy. Canadian Perinatal Health Report. Ottawa: Health Canada; 2008; 39-42.

5. US Department of Health and Human Services. Health consequences of tobacco use among women: reproductive outcomes. Women and Smoking. Washington DC: Office of the Surgeon General 2001; 272-307.

6. England LJ, Kendrick JS, Gargiullo PM, Zahniser SC, Hannon WH. Measures of maternal tobacco exposure and infant birth weight at term. Am J Epidemiol 2001; 153: 954-60.

7. Vardavas Cl, Chatzi L, Patelarou E, et al. Smoking and smoking cessation during early pregnancy and its effect on adverse pregnancy outcomes and fetal growth. Eur J Pediatr 2010; 169: 741-8.

8. Salmasi G, Grady R, Jones J, McDonald SD; Knowledge Synthesis Group. Environmental tobacco smoke exposure and perinatal outcomes: a systematic review and meta-analyses. Acta Obstet Gynecol Scand 2010; 89: 423-41.

9. Lindbohm ML, Sallmén M, Taskinen H. Effects of exposure to environmental tobacco smoke on reproductive health. Scand J Work Environ Health 2002; 28 Suppl 2: 84-96.

10. Kharrazi M, DeLorenze GN, Kaufman FL, et al. Environmental tobacco smoke and pregnancy outcome. Epidemiology 2004; 15: 660-70.

11. Windham GC, Eaton A, Hopkins B. Evidence for an association between environmental tobacco smoke exposure and birthweight: a meta-analysis and new data. Paediatr Perinat Epidemiol 1999; 13: 35-57.

12. US Department of Health and Human Services. The health consequences of smoking: nicotine addiction. A report of the Surgeon General. Rockville: US Government Printing Office 1988; 26-50. 
13. Shah CP. Public Health and Preventative Medicine in Canada. $5^{\text {th }}$ ed. Elsevier, Toronto 2003; 201.

14. Bergen HT. Exposure to smoke during development: fetal programming of adult disease. Tob Induc Dis 2006; 3: 5-16.

15. Werler MM. Teratogen update: smoking and reproductive outcomes. Teratology 1997; 55: 382-8.

16. Mantzoros CS, Varvarigou A, Kaklamani VG, Beratis NG, Flier JS. Effect of birth weight and maternal smoking on cord blood leptin concentrations of full-term and preterm newborns. J Clin Endocrinol Metab 1997; 82: 2856-61.

17. PRAMS. Center for Disease Control and Prevention. http:// www.cdc.gov/prams. Accessed November 19, 2017.

18. Wojtyla C, Wojtyla-Buciora P. Polish Pregnancy-related Assessment Monitoring System (Pol-PrAMS): research on lifestyle health behaviors of Polish women during gestation - study design. J Health Inequal 2016; 2: 185-91.

19. Wojtyła C, Wojtyła-Buciora P. Cigarette smoking among pregnant women in Poland. J Health Inequal 2017; 3: 47-50.

20. Kramer MS. Determinants of low birth weight: methodological assessment and meta-analysis. Bull World Health Organ 1987; 65: 663-737.

21. Jaddoe VW, Troe EJ, Hofman A, et al. Active and passive maternal smoking during pregnancy and the risks of low birthweight and preterm birth: the Generation R Study. Paediatr Perinat Epidemiol 2008; 22: 162-71.

22. Miyake Y, Tanaka K, Arakawa M. Active and passive maternal smoking during pregnancy and birth outcomes: the Kyushu Okinawa maternal and child health study. BMC Pregnancy Childbirth 2013; 13: 157.

23. Chełchowska M, Gajewska J, Mazur J, Ambroszkiewicz Maciejewski TM, Leibschang J. Serum pregnancy-associated plasma protein A levels in the first, second and third trimester of pregnancy: relation to newborn anthropometric parameters and maternal tobacco smoking. Arch Med Sci 2016; 12: 1256-62.

24. Bernstein IM, Mongeon JA, Badger GJ, Solomon L Heil SH, Higgins ST. Maternal smoking and its association with birth weight. Obstet Gynecol 2005; 106: 986-91.

25. McCowan LM, Dekker GA, Chan E, et al. Spontaneous preterm birth and small for gestational age infants in women who stop smoking early in pregnancy: prospective cohort study. BMJ 2009; 338: b1081.

26. Leonardi-Bee J, Smyth A, Britton J, Coleman T. Environmental tobacco smoke and fetal health: systematic review and meta-analysis. Arch Dis Child Fetal Neonatal 2008; 93: F351-61.

27. Pogodina C, Brunner Huber LR, Racine EF, Platonova E. Smoke-free homes for smoke-free babies: the role of residential environmental tobacco smoke on low birth weight. J Community Health 2009; 34: 376-82.

28. Office on Smoking and Health (US). The Health Consequences of Involuntary Exposure to Tobacco Smoke: A Report of the Surgeon General. Atlanta (GA): Centersfor Disease Control and Prevention (US); 2006.

29. Haddow JE, Knight GJ, Palomaki GE, Kloza EM, Wald NJ. Cigarette consumption and serum cotinine in relation to birth weight. Br J Obstet Gynaecol 1987; 94: 678-81.

30. Pickett KE, Rathouz PJ, Kasza K, Wakschlag LS, Wright R. Self-reported smoking, cotinine levels, and patterns of smoking in pregnancy. Paediatr Perinat Epidemiol 2005; 19: 368-76. 